\title{
Risk Stratification in Hypertrophic Cardiomyopathy
}

\author{
Alexandros Klavdios Steriotis and Sanjay Sharma
}

CRY Centre for Inherited Cardiovascular Conditions \& Sports Cardiology, St George's University of London, London, UK

\begin{abstract}
Hypertrophic cardiomyopathy (HCM) is a hereditary primary myocardial disease that is most commonly due to mutations within genes encoding sarcomeric contractile proteins and is characterised by left ventricular hypertrophy in the absence of a cardiac or systemic cause. Although the overall prognosis is relatively good with an annual mortality rate $<1 \%$, the propensity to potentially fatal ventricular arrhythmias is the most feared complication. The identification of patients at risk of arrhythmogenic sudden cardiac death (SCD) is an essential component in disease management. Aborted SCD and malignant ventricular arrhythmias are the most powerful risk factors for SCD and ICD implantation is recommended in such circumstances. The selection of patients who may benefit from ICD therapy for primary prevention purposes is more challenging. The heterogeneous nature of the disease and the variation in trigger factors provides an adequate explanation for the low predictive accuracy of most conventional risk factors in isolation. A new risk model for risk stratification proposed by the European Society of Cardiology HCM outcome group shows promise but requires validation in different cohorts. The ICD is the only effective therapy in preventing SCD for the disease with a relatively low adverse event rate, but most deaths occur in relatively young patients. However, it is also difficult to ignore the complications with the ICD, therefore, the strive to perfect risk stratification in HCM should continue to ensure that only the most high-risk patients receive an ICD.
\end{abstract}

\section{Keywords}

Hypertrophic cardiomyopathy, risk stratification, sudden cardiac death

Disclosure: The authors have no conflicts of interest to declare.

Received: 22 May 2015 Accepted: 19 June 2015 Citation: European Cardiology Review, 2015;10(1):31-6

Correspondence: Sanjay Sharma, St George's University of London, Cranmer Terrace, London, SW17 ORE, UK. E: sasharma@sgul.ac.uk

Hypertrophic cardiomyopathy ( $\mathrm{HCM}$ ) is a hereditary primary myocardial disease that is most commonly caused by mutations within genes encoding sarcomeric contractile proteins and is characterised by left ventricular hypertrophy in the absence of a cardiac or systemic cause. ${ }^{1,2}$ The condition is inherited as an autosomal dominant trait and has a prevalence of one in 500.3.4 Marked genetic heterogeneity, diverse clinical phenotypes and a highly variable natural history are well recognised. ${ }^{4-6}$ Although the overall prognosis is relatively good with an annual mortality rate $<1 \%$, the propensity to potentially fatal ventricular arrhythmias is the most feared complication particularly because the peak incidence of sudden cardiac death (SCD) is during adolescence and early adulthood. ${ }^{6-9}$ The association with SCD is most frequently highlighted when a young, previously asymptomatic athlete falls victim and HCM is considered the commonest cause of SCD in young athletes worldwide. ${ }^{10-12}$

The arrhythmogenic substrate comprises left ventricular hypertrophy, myocyte disarray and interstitial fibrosis. ${ }^{13-17}$ Triggers for arrhythmias may include myocardial ischaemia, excessive sympathetic stimulation, left ventricular outflow tract obstruction (LVOTO) and paroxysmal atrial fibrillation (AF). ${ }^{18-20}$ The identification of patients at risk of arrhythmogenic SCD is an essential component in disease management given that the implantable cardioverter defibrillator (ICD) is the most effective therapy in preventing SCD. ${ }^{21-25}$ However, the low risk of adverse events in most patients coupled with the complex and unpredictable relationship between the arrhythmic substrate and triggers for arrhythmias means that risk stratification for arrhythmogenic SCD is a challenging aspect of the disease. A low threshold to implant an ICD into most patients with $\mathrm{HCM}$ is not cost-effective and is hampered by the high prevalence of inappropriate shocks and other complications relating to the implantation of the ICD. ${ }^{23-26}$

Aborted SCD and malignant ventricular arrhythmias are the most powerful risk factors for SCD. 27-30 Patients who survive an episode of ventricular tachycardia (VT) or ventricular fibrillation (VF) remain at high risk of recurrent arrhythmogenic events, having an estimated risk of $10.6 \%$ per annum and both the American College of Cardiology Foundation/ American Heart Association (ACCF/AHA) and American College of Cardiology/European society of Cardiology (ACC/ESC) management guidelines for HCM recommend ICD implantation in such patients. ${ }^{27-30}$

\section{Conventional Risk Factors for SCD in HCM}

The selection of patients who may benefit from ICD therapy for primary prevention purposes is more challenging. Several potential risk factors for SCD have been reported, however conventionally regarded major risk factors include unexplained syncope, family history of sudden cardiac death (FHSCD), severe left ventricular hypertrophy (LVH), non-sustained VT (NSVT) on the Holter-monitoring or during exercise and an abnormal blood pressure response to exercise (ABPRE) (see Table 1) ${ }^{19,28-32}$ Consistent with the clinical diversity of the disease, all of these risk factors have a relatively low positive predictive accuracy in the range of $20 \% .{ }^{29}$ Conversely, each factor has an excellent negative predictive accuracy therefore a patient who does not exhibit any of these risk factors is suitably deemed low risk. ${ }^{29,31}$ Nevertheless, up to 
Table 1: Major SCD Risk Factors and Modifiers Used in the Current Guidelines and Putative Risk Factors Described in the Literature, but not Included in the Current Guidelines

\begin{tabular}{|c|c|}
\hline $\begin{array}{l}\text { Major SCD Risk Factors } \\
\text { (2003 ACC/ESC and } 2011 \text { ACCF/AHA Guideli }\end{array}$ & References \\
\hline Family history of sudden cardiac death & $37,41,54$ \\
\hline Unexplained syncope & $37,38,42,43,54$ \\
\hline Severe left ventricular hypertrophy & $33,36,44,45,52,54$ \\
\hline Non-sustained ventricular tachycardia & $33,37,47,48,54$ \\
\hline Abnormal blood pressure response to exercise & $33,35,50,51,54$ \\
\hline \multicolumn{2}{|c|}{ Potential SCD Risk Modifiers (2011 ACCF/AHA Guidelines) } \\
\hline Left ventricular outflow tract obstruction & $32,51-54$ \\
\hline Late Gadolinium enhancement on CMR Imaging & $60,62,63,70$ \\
\hline Genetic Mutations & $76-83$ \\
\hline Left ventricular apical aneurysm & 84 \\
\hline End-stage phase of $\mathrm{HCM}(\mathrm{EF}<50 \%)$ & 85,86 \\
\hline \multicolumn{2}{|c|}{ Additional SCD Risk Factors (2014 ESC Guidelines)* } \\
\hline Increased left atrial diameter & 9,38 \\
\hline Young age at the evaluation & 9, 36-39 \\
\hline \multicolumn{2}{|l|}{ Putative SCD Risk Factors } \\
\hline Paced ventricular electrogram fractionation & 40 \\
\hline QRS fragmentation on the ECG & 87,88 \\
\hline Exercise-induced NSVT/VF & 47 \\
\hline Severe obstructive coronary disease & 92 \\
\hline Microvascular ischaemia & $94-96$ \\
\hline Midventricular obstruction & 89,90 \\
\hline Atrial fibrillation & 28,92 \\
\hline
\end{tabular}

*The 2014 European Society of Cardiology (ESC) guidelines considered as sudden cardiac death (SCD) risk factors: left atrial diameter, age, family history of $S C D$, unexplained syncope, left ventricular outflow tract obstruction (LVOTO), severe left ventricular hypertrophy and non-sustained ventricular tachycardia (NSVT) and excluded the abnormal blood pressure response to exercise. $A C C=$ American College of Cardiology; $A C C=$ American college of Cardiology Foundation; $A H A=$ American Heart Association; $C M R=$ cardiovascular magnetic resonance; $E C G$ = electrocardiogram; $E F=$ ejection fraction; $H C M=$ hypertrophic cardiomyopathy; $\mathrm{VF}=$ ventricular fibrillation

$3 \%$ of arrhythmogenic SCDS occur in patients who do not exhibit any of these risk factors. ${ }^{33,34}$

The significance of these risk factors is governed by age..$^{32-39}$ In young patients, syncope, severe LVH and NSVT are particularly associated with an increased risk of SCD. ${ }^{30,35-39}$ In older patients who have survived more than 60 years, the risk of arrhythmogenic SCD is low despite the presence of the five conventional risk factors above. ${ }^{39}$

Invasive electrophysiological studies, such as programmed ventricular stimulation, have a poorer predictive accuracy than some of the risk factors mentioned above and is not indicated for risk stratification. ${ }^{30}$ Paced ventricular electrogram fractionation analysis has been reported to reveal a positive predictive accuracy in the range of $38 \% .{ }^{40}$ However, the invasive nature of the procedure in combination with the dynamic nature of the risk profile of SCD in HCM patients means that periodical assessment is impractical.

\section{Family History of SCD}

A FHSCD from HCM in first-degree relatives of an affected patient or the presence of one or more premature SCD in the family has always been considered to represent an important risk factor because it is recognised that SCD events often cluster in families. ${ }^{29}$ Patients receiving an ICD for primary prevention based on a family history of HCM-related SCD experience appropriate electrical discharges comparable to other patient subsets with high-risk markers. ${ }^{41}$

\section{Unexplained Syncope}

Determination of the possible cause of unwitnessed syncope is challenging in HCM because there are multiple potential causes that include vasovagal syncope, arrhythmogenic syncope, abnormal vascular responses or transient severe mechanical LVOTO. ${ }^{28}$ However, the clinical perception is that syncope is the only premonitory cardiac symptom that is associated with SCD. ${ }^{28,42,43}$ Patients with syncopal events that occur in close temporal proximity (6 months) to the initial evaluation, show a substantially higher risk of SCD than patients without syncope. ${ }^{38}$ Older patients with remote syncopal events do not show an increased risk. ${ }^{38}$

\section{Severe Left Ventricular Hypertrophy}

The severity and extent of LVH is associated with increased risk of SCD. ${ }^{30}$ Several studies have shown that a maximum wall thickness of $\geq 30 \mathrm{~mm}$ is associated with greatest risk of SCD. ${ }^{44-46}$ The ACCF/AHA guidelines state that the presence of extreme LVH alone is reasonable to recommend ICD ${ }^{29}$ (see Figure 1); however, extreme LVH is relative rare and the maximum wall thickness of a single segment may not adequately represent the true burden of hypertrophy. ${ }^{19}$ According to the 2003 ESC guidelines, the degree of maximum left ventricle (LV) wall thickness should be considered in the context of a multifactorial approach to risk stratification, rather than as an isolated risk factor. ${ }^{20,36}$ An exception may be the development of severe $\mathrm{LVH}$ at a young age $\left(<18\right.$ years). ${ }^{36}$

\section{Non-sustained Ventricular Tachycardia}

The presence of repetitive ventricular arrhythmias, at rest or effortinduced is frequently used as a marker of increased electrical instability of the myocardium in clinical practice., ${ }^{37,47}$ NSVT (defined as $\geq 3$ consecutive beats with a heart rate of $\geq 120 \mathrm{bpm}$ ) is detected in approximately $20 \%$ of HCM patients and is associated with a substantial increase in SCD risk in young patients aged $\leq 30$ years old. ${ }^{37}$ A relationship between the frequency, duration and rate of NSVT episodes has not yet been clearly demonstrated. ${ }^{37}$ In clinical practice isolated brief runs of NSVT on random Holter-monitoring rarely trigger decisions for prophylactic ICD, whereas frequent and/or prolonged ( $>10$ beats) bursts of NSVT identified over serial monitoring periods, intuitively carry greater weight as a risk factor. ${ }^{48}$ In one study of $104 \mathrm{HCM}$ patients with an ICD the presence of NSVT was the most predictive risk factor for appropriate ICD discharge in the 78 patients of the primary prevention group..$^{24}$

\section{Abnormal Blood Pressure Response to Exercise}

Approximately one-third of patients with $\mathrm{HCM}$, have an ABPRE (defined as either the failure to increase by at least $20 \mathrm{mmHg}$ or a drop of at least $20 \mathrm{mmHg}$ during effort), which can be due to central and peripheral mechanisms. ${ }^{28,29,49}$ An ABPRE rarely represents the sole indication for a prophylactic ICD implant in clinical practice and is usually considered in association with other risk factors (see Figure 1).29,35 The 2014 ESC guidelines did not consider the ABPRE as a risk factor since it has not been independently associated with SCD in any multivariate survival analysis (see Figure 2). ${ }^{29,30,50,51}$

\section{Potential SCD Modifiers in HCM according to 2011 ACCF/AHA guidelines}

Left Ventricular Outflow Tract Obstruction at Rest

Dynamic LVOTO is reported in approximately $25 \%$ of patients during resting conditions. ${ }^{52,53} \mathrm{~A}$ study on 917 patients, including almost one- 
Figure 1: Flow Chart of the 2011 ACCF/AHA Model for ICD Implantation for Primary Prevention

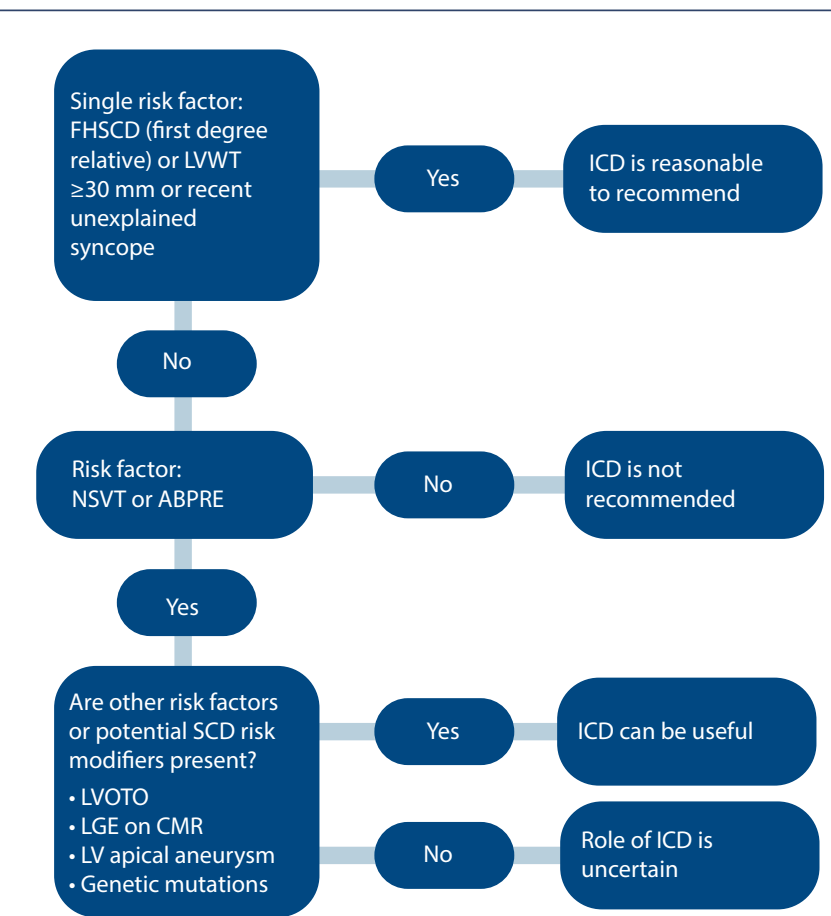

$A B P R E=$ abnormal blood pressure response to exercise; $C M R=$ cardiovascular magnetic resonance; $F H S C D$ = family history of sudden cardiac death $(S C D) ; I C D=$ implantable cardioverter defibrillator; $L G E$ = late Gadolinium enhancement; $L V O T O=$ left ventricular outflow tract obstruction; $L W W T=$ left ventricular wall thickness; NSVT = non-sustained ventricular tachycardia.

third with LVOTO, demonstrated an association between LVOTO and increased risk of SCD and appropriate ICD discharges over a 61-month median follow-up period..$^{54}$ The risk of SCD was related to the severity of LVOTO and the presence of other recognised risk factors for SCD. Multivariable analysis demonstrated that LVOTO was an independent predictor of SCD/ICD discharge, with a 2.4-fold increase in the risk of SCD/ICD discharge. ${ }^{54}$ The role of provocable LVOTO with exercise is unclear and current guidelines do not recommend exercise-induced LVOTO in the risk stratification. ${ }^{30,55}$

\section{Late Gadolinium Enhancement on CMR Study}

The past decade has witnessed a burgeoning in the number of articles relating to the role of cardiovascular magnetic resonance

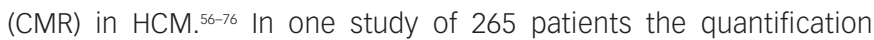
of LV mass correlated weakly with maximal wall thickness and was $100 \%$ sensitive in predicting HCM-related mortality, but had a specificity of just $39 \% .^{56}$ However, most of the interest in CMR is focused on the late enhancement after Gadolinium. ${ }^{57-72}$ Late Gadolinium enhancement (LGE) probably constitutes areas of myocardial replacement fibrosis and is detected in up to 60-70\% of HCM patients. ${ }^{57-63}$ As with all other aspects of the disease there is considerable heterogeneity in the extent and pattern of LGE. ${ }^{57-63}$ Fibrotic remodelling occurs early in disease pathogenesis of HCM but it may also be a secondary phenomenon related to microvascular ischaemia. ${ }^{13-17,64-66}$ Fibrous tissue represents a principal substrate for re-entrant ventricular arrhythmias and contributes to increased ventricular stiffness. ${ }^{59} \mathrm{~A}$ few studies have reported that the presence of LGE is significantly associated with heart failure death and all-cause mortality and is an independent predictor of adverse outcome and disease progression..$^{59-62}$ In one study of 217 HCM patients the presence of fibrosis was associated with a 3.4-fold risk of major adverse events and the risk was proportional to the extent of
Figure 2: Flow Chart of 2014 ESC Model for ICD Implantation for Primary Prevention

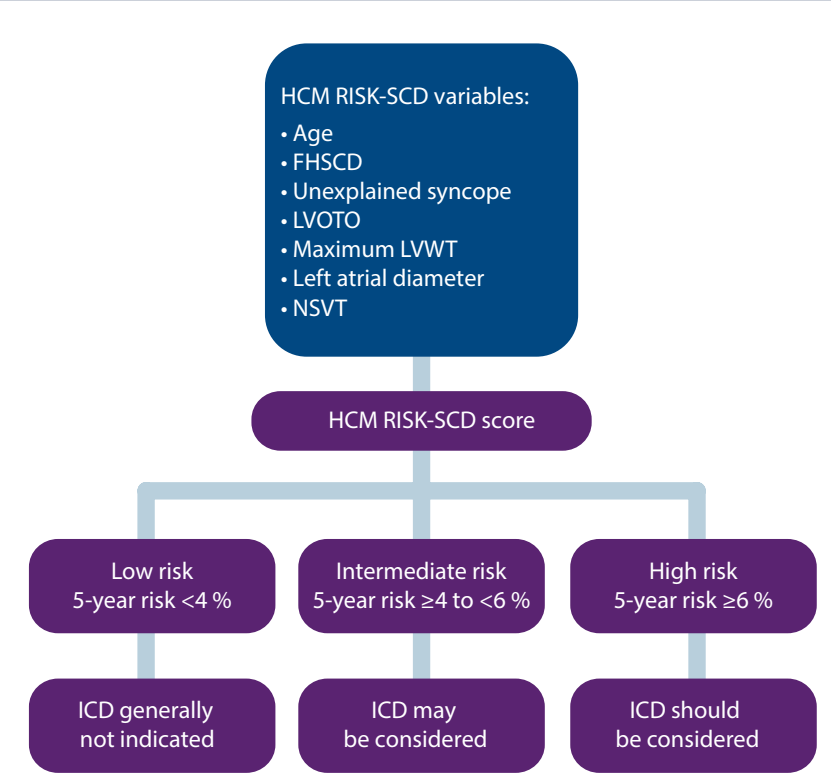

$A B P R E=$ abnormal blood pressure response to exercise; $C M R=$ cardiovascular magnetic resonance; $F H S C D$ = family history of sudden cardiac death $(S C D)$; ICD = implantable cardioverter defibrillator; $L G E=$ late Gadolinium enhancement; $L V=$ left ventricle; LVOTO = left ventricular outflow tract obstruction; LVWT = left ventricular wall thickness; NSVT = non-sustained ventricular tachycardia.

LGE. ${ }^{59}$ Another study of $177 \mathrm{HCM}$ patients showed that the presence of LGE may identify patients with increased susceptibility to ventricular tachyarrhythmias on the ambulatory Holter-monitoring (including a sevenfold increase in the risk of NSVT) and even small areas of LGE may be sufficient to promote arrhythmias. ${ }^{68}$ The significance of LGE in predicting arrhythmogenic SCD remains controversial. A recent metanalysis of four studies evaluating 1,053 patients, over an average follow-up of 3.1 years, concluded that LGE shows a trend towards significance for predicting $S C D$, but failed to shown a significant independent association. ${ }^{0}$ The high prevalence of LGE in HCM patients means that it would be impractical to consider it as a risk factor for SCD in isolation although extensive LGE has been shown to be associated with progressive ventricular dilatation and heart failure. $58,59,69$ Recently, two studies have provided conflicting results regarding the value of the extensive LGE in risk stratification for SCD.63,70 One study included $711 \mathrm{HCM}$ patients, with a median follow-up of 3.5 years and $66 \%$ of patients had LGE. ${ }^{63}$ The extent of LGE was found to be a strong univariable predictor of SCD, which was not maintained after adjustment for LV ejection fraction. ${ }^{63}$ The other study included 1,293 HCM patients, with a median follow-up of 3.3 years and presence of LGE in $42 \%$ of patients. SCD events occurred in 37 patients (3\%), including 17 (1.3\%) with appropriate ICD discharge, which was considered equivalent to SCD. ${ }^{70}$ The extent of LGE was associated with an increased risk of SCD events and in particular LGE $\geq 15 \%$ of the LV mass demonstrated a twofold increase in SCD event rate in those patients who would otherwise considered be at low risk. The authors concluded that extensive LGE provided additional information for assessing SCD event risk, particularly in HCM patients otherwise judged to be at low risk..$^{70}$ The major criticism of this study was that even if the statistical analysis appeared to support this statement, the raw data did not. ${ }^{11}$ In the 20 patients that died suddenly or experienced aborted SCD only one revealed extensive LGE, while in the 17 patients experiencing an appropriate ICD shock, 13 patients had recognised conventional risk factors and 
from the rest only three had extensive LGE. ${ }^{70}$ In conclusion, the extent of LGE on CMR has some utility in predicting cardiovascular mortality, but the current data are contradictory and not conclusive in order to support the use of LGE in predicting the risk of arrhythmogenic SCD. ${ }^{30,71}$ Newer CMR techniques (as T1-mapping) may improve the characterisation of myocardial substrate of the arrhythmias..$^{72-75}$

\section{Additional Role of Genetics}

Most sarcomere mutations capable of causing HCM are novel and limited to individual families; therefore, genetic screening is of limited value in risk stratification in most cases. $4,5,29,30,76,77$ The presence of multiple mutations or specific mutations encoding troponin T and lysosomalassociated membrane protein-2 (LAMP-2) may be indicative of a high risk of fatal events. ${ }^{78-83}$

\section{Specific Cases}

\section{Left Ventricular Aneurysm}

Left ventricular apical aneurysm with regional scarring is considered as a potential risk factor for primary prevention and has recently been reported in a high-risk subset of HCM patients. ${ }^{84}$ Left ventricular apical aneurysm is rare (approximately in $2 \%$ of HCM patients) and is best characterised by CMR imaging. In one study of 28 patients, almost half of the patients with left ventricular apical aneurysm experienced adverse disease complications (event rate $10.5 \%$ /year), including SCD, appropriate ICD discharges, non-fatal thromboembolic stroke and progressive heart failure and death. ${ }^{84}$

\section{End-stage Phase of $\mathrm{HCM}$}

End-stage phase of HCM affects 3-8\% of individuals and is characterised by progressive thinning of the myocardium with cavity enlargement and impaired systolic function. ${ }^{85,86}$ The complication is a result of extensive and transmural fibrosis and has a high incidence of SCD with an annual mortality rate exceeding $10 \% .85 .86$ In such patients, prophylactic ICD implantation is a generally accepted clinical practice. ${ }^{29,85,86}$

\section{ACC/ESC Guidelines versus 2011 ACCF/AHA Guidelines}

The main disagreement between the US and Europe guidleines is historically based on the number of risk factors required before consideration of an implantation of an ICD for primary prevention..$^{28,29}$ Given the low positive predictive value of each of the conventional risk factors, the European approach has been to implant an ICD only in the presence of $>1$ risk factor. ${ }^{28}$ By contrast, the US approach recommends ICD implantation patients with FHSCD from HCM in a first-degree relative, LV wall thickness $\geq 30 \mathrm{~mm}$ or recent unexplained syncope as isolated risk factors whereas those patients with NSVT or an ABPRE require another risk factor or risk modifier (such as LVOTO, LGE on CMR imaging, LV apical aneurysm or a high-risk genetic mutation ${ }^{29}$ (see Figure 1). This difference in approach is partially due to the conflicting results between American and European studies regarding the risk stratification. ${ }^{22,33}$ Previous American studies have reported that an important proportion of discharges occur in patients implanted with a prophylactic ICD with just one risk factor. ${ }^{22}$ The European concern is that if ICDS were inserted in all patients with one risk factor the incidence of device complications would surpass the potential benefits. ${ }^{33,34}$ Although there is no doubt about the value of an ICD in preventing SCD with appropriate discharge rates ranging from 2-3.6 \%/year for primary prevention cases and 4.3-10.6\%/ year for secondary prevention cases, the inappropriate shock rate and implant complications range from $16-27 \%$ and $12-18 \%$, respectively. ${ }^{22,23} \mathrm{~A}$ recent meta-analysis of $16 \mathrm{HCM}$ cohorts reported inappropriate ICD interventions and complication of $4.8 \% / y e a r$ and $3.4 \% /$ year, respectively. 25

\section{HCM Risk-SCD Model of the 2014 ESC Guidelines}

A fundamental problem with the aforementioned risk stratification procedure is the assumption that the significance of all of the risk factors remains static throughout life. Furthermore, important parameters such as LVH or LVOTO, which are contiguous variables, are treated as binary factors (present or absent). The ESC HCM outcome investigators have recently recommended a 5-year risk calculator derived from a model involving a large retrospective longitudinal multicentre experience from 3,675 patients.9.30 Eight clinical parameters were included as pre-specified predictors that were independently associated with SCD in at least one published study of multivariate survival analysis. Of the eight parameters, seven were associated with SCD or an appropriate ICD shock at the $15 \%$ significance level and these were: age, FHSCD, maximal LV wall thickness, left atrial diameter, maximal LVOTO, NSVT and unexplained syncope (see Figure 2).

The incorporation of these parameters into the model equation is used to estimate the 5-year risk of SCD for any particular patient. The cut-off level of $\geq 6 \%$ SCD risk in 5 years is recommended for considering an ICD implant for primary prevention. Individuals with a risk score of $<4 \%$ are considered as low risk, whereas those with a risk score of 4-6\% of SCD characterise an intermediate-risk group where ICD may be considered (see Figure 2).

The results of this study indicate that the use of this model is superior to prior conventional methods for detecting high-risk individuals previously considered at low or intermediate risk. The model also scores highly for correctly identifying individuals at high risk of SCD. ${ }^{30}$

The new 2014 ESC risk stratification model is limited to some extent in that it was not validated in paediatric patients ( $<16$ years), in patients with syndromic LVH or in a large population of non-Caucasian individuals. Furthermore the effect of latent LVOTO or the effect of LVOTO reduction by alcohol ablation or myectomy was not tested and very few patients had extreme $\mathrm{LVH} \geq 35 \mathrm{~mm} .{ }^{30}$

\section{Other Potential Risk Factors and Arbitrators not Included in the Current Guidelines}

A number of electrical, structural and functional markers that can be assessed using simple investigations have been proposed for risk stratification. The fragmentation of the QRS on the ECG has been postulated to predict ventricular arrhythmic events. ${ }^{87,88}$ A study in 167 patients with a mean follow-up of 6.3 years, fragmentation of the QRS was a strong independent predictor for major arrhythmic events including SCD. ${ }^{87}$

There are reports that HCM associated with midventricular obstruction (with pressure gradient $\geq 30 \mathrm{mmHg}$ ) may be an independent predictor of adverse outcomes, especially the combined endpoint of SCD and potentially lethal arrhythmic events..$^{89,90}$ Conversely, apical HCM has been associated with a benign prognosis. ${ }^{91}$

Myocardial ischaemia that may be caused by small vessel disease or concomitant severe epicardial coronary artery disease has also been considered as possible risk factor. ${ }^{28,92,93}$ In one study of $433 \mathrm{HCM}$ patients, $27 \%$ had severe epicardial CAD and this was a significant predictor 
for cardiac death and SCD. ${ }^{92}$ Assessment of coronary microvascular dysfunction is challenging and stress perfusion CMR imaging could have a future role in risk stratification. ${ }^{94-96}$ Exercise capacity has also been proposed to help risk stratification in different studies. ${ }^{97-99}$ it has been demonstrated that peak VO2 is associated with an increased risk of major events during short-term follow-up.99

In conclusion, there have been significant advances in the risk stratification of HCM since the disease was first described over 5 decades ago. The heterogeneous nature of the disease and the variation in trigger factors provides an adequate explanation for the low predictive accuracy of most conventional risk factors in isolation. A new risk model for risk stratification proposed by the ESC HCM outcome group shows promise but requires validation in different cohorts. The ICD is the only effective therapy in preventing SCD for the disease with a relatively low adverse event rate, but most deaths occur in relatively young patients. However, it is also difficult to ignore the complications with the ICD, therefore, the strife to perfect risk stratification in $\mathrm{HCM}$ should continue to ensure that only the most high-risk patients receive an ICD.
1. Elliott $P$, Andersson B, Arbustini E, et al., Classification of the cardiomyopathies: a position statement from the European Society Of Cardiolos Pericardial Diseases, Eur Heart J, 2008:29:270-6.

2. Maron BJ, Towbin JA, Thiene G, et al., American Heart Association; Council on Clinical Cardiology, Heart Failure and Transplantation Committee; Quality of Care and Outcomes Research and Functional Genomics and Translational Biology Interdisciplinary Working Groups; Council on Epidemiology and Prevention. Contemporary definitions and classification of the cardiomyopathies: an American Heart Association scientific Statement from the Council on Clinical Cardiology, Heart Failure and Transplantation Committee; Quality of and Translational Biology Interdisciplinary Working Groups; and Translational Biology Interdisciplinary Working Groups; and Council on Epider

3. Maron BJ, Gardin JM, Flack JM, et al., Prevalence of hypertrophic cardiomyopathy in a general population of young adults. Echocardiographic analysis of 4111 subjects in the CARDIA Study. Coronary Artery Risk Development in (Young) Adults, Circulation, 1995;92:785-9.

4. Ackerman MJ, Priori SG, Willems S, et al., HRS/EHRA exper consensus statement on the state of genetic testing for the channelopathies and cardiomyopathies, Europace, 2011:13:1077-109.

5. Charron P, Arad M, Arbustini E, et al., Genetic counselling and testing in cardiomyopathies: a position statement of the European Society of Cardiology Working Group on Myocardia and Pericardial Diseases, Eur Heart J, 2010;31:2715-26.

6. Cecchi F, Olivotto I, Montereggi A, Santoro G, et al., Hypertrophic cardiomyopathy in Tuscany: clinical course and outcome in an unselected regional population, J Am Coll Cardiol, 1995;26:1529-36.

7. Maron BJ, Olivotto I, Spirito P, et al., Epidemiology of hypertrophic cardiomyopathy-related death: revisited in a large non-referral-based patient population, Circulation, 2000:102:858-64

8. Elliott PM, Gimeno JR, Thaman R, et al., Historical trends in reported survival rates in patients with hypertrophic cardiomyopathy, Heart, 2006;92:785-91.

9. O'Mahony C, Jichi F, Pavlou M, et al., Hypertrophic Cardiomyopathy Outcomes Investigators. A novel clinical risk prediction model for sudden cardiac death in hypertrophic cardiomyopathy (HCM risk-SCD), Eur Heart 」, 2014:35:2010-20.

10. Maron BJ, Shirani J. Poliac LC, et al. Sudden death in young competitive athletes. Clinical, demographic, and pathologica profiles, JAMA, 1996:276:199-204.

11. Corrado D, Basso C, Schiavon M, et al., Screening fo hypertrophic cardiomyopathy in young athletes, N Eng/ J Med 1998;339:364-9.

12. de Noronha SV, Sharma S, Papadakis M, et al., Aetiology of sudden cardiac death in athletes in the United Kingdom: pathological study, Heart, 2009;95:1409-14.

13. Shirani J, Pick R, Roberts WC, et al., Morphology and significance of the left ventricular collagen network in young patients with hypertrophic cardiomyopathy and sudden cardiac death, J Am Coll Cardiol, 2000:35:36-44.

14. Varnava AM, Elliott PM, Sharma S, et al., Hypertrophic cardiomyopathy: the interrelation of disarray, fibrosis, and small vessel disease, Heart, 2000;84:476-82.

15. Varnava AM, Elliott PM, Mahon N, et al., Relation between myocyte disarray and outcome in hypertrophic cardiomyopathy, Am I Cardiol, 2001;88:275-9.

16. Maron BJ, Wolfson JK, Epstein SE, et al., Intramural ("small vessel") coronary artery disease in hypertrophic cardiomyopathy, I Am Coll Cardiol, 1986:8:545-57.

17. Basso C Thiene G Corrado D, et al, Hypertrophic cardiomyopathy and sudden death in the young: pathologic evidence of myocardial ischemia, Hum Pathol, 2000;31:988-98.

18. Cha YM, Gersh BJ, Maron BJ, et al., Electrophysiologic manifestations of ventricular tachyarrhythmias provoking appropriate defibrillator interventions in high-risk patients with hypertrophic cardiomyopathy, I Cardiovasc Electrophysiol 2007; 18:483-7.

19. O'Mahony C, Elliott PM. Prevention of sudden cardiac death in hypertrophic cardiomyopathy, Heart, 2014;100:254-60

20. Sen-Chowdhry S, MCKenna WJ, Sudden death from genetic and acquired cardiomyopathies, Circulation, 2012:125:1563-76. 21. Maron BJ, Shen WK, Link MS, et al., Efficacy of implantable cardioverter-defibrillators for the prevention of sudden death in patients with hypertrophic cardiomyopathy, N Eng/ I Med, 2000;342:365-73.

22. Maron BJ, Spirito $P$, Shen WK, et al., Implantable cardioverterdefibrillators and prevention of sudden cardiac death in hypertrophic cardiomyopathy, JAMA, 2007;298:405-12.

23. O'Mahony C, Lambiase PD, Quarta G, et al., The longterm survival and the risks and benefits of implantable cardioverter defibrillators in patients with hypertrophic cardiomyopathy, Heart, 2012;98:116-25.

24. Syska P, Przybylski A, Chojnowska L, et al., Implantable cardioverter-defibrillator in patients with hypertrophic cardiomyopathy: efficacy and complications of the therapy in long-term follow-up, J Cardiovasc Electrophysiol, 2010:21:883-9.

25. Schinkel AF Vriesendorp PA, Sijbrands EJ, et al., Outcome and complications after implantable cardioverter defibrillato therapy in hypertrophic cardiomyopathy: systematic review and meta-analysis, Circ Heart Fail, 2012;5:552-9.

26. Lin G, Nishimura RA, Gersh BJ, et al., Device complication and inappropriate implantable cardioverter defibrillator shocks in patients with hypertrophic cardiomyopathy, Heart, 2009:95:709-14.

27. Elliott PM, Sharma S, Varnava A, et al., Survival after cardiac arrest or sustained ventricular tachycardia in patients with hypertrophic cardiomyopathy, I Am Coll Cardiol, 1999;33:1596-601

28. Maron BJ, McKenna WJ, Danielson GK, et al., American College of Cardiology Foundation Task Force on Clinical Expert Consensus Documents; European Society of Cardiology Committee for Practice Guidelines. American College of Cardiology/European Society of Cardiology Clinical Expert Consensus Document on Hypertrophic Cardiomyopathy. A report of the American College of Cardiology Foundation Task Force on Clinical Expert Consensus Documents and the European Society of Cardiology Committee for Practice Guidelines, Eur Heart J, 2003:24:1965-91.

29. Gersh BJ, Maron BJ, Bonow RO, et al., 2011 ACCF/AHA guideline for the diagnosis and treatment of hypertrophic cardiomyopathy: a report of the American College of Cardiology Foundation/American Heart Association Task Force on Practice Guidelines, Circulation, 2011;124:e783-831.

30. Elliott PM, Anastasakis A, Borger MA et al., 2014 ESC Guidelines on diagnosis and management of hypertrophic cardiomyopathy: the Task Force for the Diagnosis and Management of Hypertrophic Cardiomyopathy of the European Society of Cardiology (ESC). Eur Heart 1 , 2014;35:2733-79.

31. O'Mahony C, Elliott P, McKenna, Sudden cardiac death in hypertrophic cardiomyopathy, Circ Arrhythm Electrophysiol, 2013;6:443-51.

32. Christiaans I, van Engelen $\mathrm{K}$, van Langen IM, et al., Risk stratification for sudden cardiac death in hypertrophic cardiomyopathy: systematic review of clinical risk markers, Europace, 2010;12:313-21

33. Elliott PM, Poloniecki J, Dickie S, et al., Sudden death in hypertrophic cardiomyopathy: identification of high risk patients, I Am Coll Cardiol, 2000:36:2212-8

34. O'Mahony C, Tome-Esteban M, Lambiase PD, et al., A validation study of the 2003 American College of Cardiology European Society of Cardiology and 2011 American College of Cardiology Foundation/American Heart Association risk stratification and treatment algorithms for sudden cardiac death in patients with hypertrophic cardiomyopathy, Heart, 2013;99:534-41

35. Olivotto I, Maron BJ, Montereggi A, et al., Prognostic value of systemic blood pressure response during exercise in a community-based patient population with hypertrophic cardiomyopathy. I Am Coll Cardiol, 1999:33:2044.

36. Olivotto I, Gistri R, Petrone P, et al., Maximum left ventricular thickness and risk of sudden death in patients with hypertrophic cardiomyopathy, I Am Coll Cardiol, 2003;41:315-21.

37. Monserrat L, Elliott PM, Gimeno JR, et al., Non-sustained ventricular tachycardia in hypertrophic cardiomyopathy: an independent marker of sudden death risk in young patients, J Am Coll Cardiol, 2003;42:873-9.

38. Spirito P, Autore C, Rapezzi C, et al., Syncope and risk of sudden death in hypertrophic cardiomyopathy, Circulation 2009;119:1703-10.

39. Maron BJ, Rowin EJ, Casey SA, et al., Risk stratification and outcome of patients with hypertrophic cardiomyopathy $\geq 60$ years of age, Circulation, 2013;127:585-93.

40. Saumarez RC, Pytkowski M, Sterlinski M, et al., Paced ventricular electrogram fractionation predicts sudden cardiac death in hypertrophic cardiomyopathy, Eur Heart s, 2008:29:1653-61

41. Bos JM, Maron BJ, Ackerman MJ, et al., Role of family history of sudden death in risk stratification and prevention of sudden death with implantable defibrillators in hypertrophic cardiomyopathy, Am I Cardiol, 2010;106:1481-6.

42. Takagi E, Yamakado T, Nakano T, Prognosis of completely asymptomatic adult patients with hypertrophic cardiomyopathy, I Am Coll Cardiol, 1999:33:206-11.

43. Kofflard MJ, Ten Cate FJ, van der Lee C, et al., Hypertrophic cardiomyopathy in a large community-based population: clinical outcome and identification of risk factors for sudden clinical outcome and identification of risk factors for sudd 2003;41:987-93.

44. Spirito P, Bellone P, Harris KM, et al., Magnitude of left ventricular hypertrophy and risk of sudden death in hypertrophic cardiomyopathy, N EngI J Med, 2000;342:1778-85.

45. Elliott PM, Gimeno Blanes JR, Mahon NG, et al., Relation between severity of left-ventricular hypertrophy and prognosis in patients with hypertrophic cardiomyopathy, Lancet, 2001;357:420-4.

46. Maron BJ, Spirito P Ackerman MJ, et al., Prevention of sudden cardiac death with implantable cardioverter-defibrillators in children and adolescents with hypertrophic cardiomyopathy, J Am Coll Cardiol, 2013;61:1527-35.

47. Gimeno JR, Tomé-Esteban M, Lofiego C, et al., Exerciseinduced ventricular arrhythmias and risk of sudden cardiac death in patients with hypertrophic cardiomyopathy, Eur Heart J, 2009;30:2599-605.

48. Spirito P, Rapezzi C, Autore C, et al., Prognosis of asymptomatic patients with hypertrophic cardiomyopathy and nonsustained ventricular tachycardia, Circulation, 1994;90:2743-7.

49. Ciampi Q, Betocchi S, Lombardi R, et al., Hemodynamic determinants of exercise-induced abnormal blood pressure response in hypertrophic cardiomyopathy, I Am Coll Cardiol, 2002;40:278-84

50. Sadoul N, Prasad K, Elliott PM, et al., Prospective prognostic assessment of blood pressure response during exercise in patients with hypertrophic cardiomyopathy, Circulation, 1997;96:2987-91

51. Maki S, Ikeda H, Muro A, et al., Predictors of sudden cardiac death in hypertrophic cardiomyopathy, Am I Cardiol, 1998:82:774-8

52. Autore $\mathrm{C}$, Bernabò $\mathrm{P}$, Barillà $\mathrm{CS}$, et al., The prognostic importance of left ventricular outflow obstruction in hypertrophic cardiomyopathy varies in relation to the severity of symptoms, J Am Coll Cardiol, 2005;45:1076-80.

53. Maron MS, Olivotto I, Betocchi S, et al., Effect of left ventricular outflow tract obstruction on clinical outcome in hypertrophic cardiomyopathy, N Eng/ J Med, 2003;348:295-303.

54. Elliott PM, Gimeno JR, Tomé MT, et al., Left ventricular outflow tract obstruction and sudden death risk in patients with hypertrophic cardiomyopathy, Eur Heart J, 2006;27:1933-41.

55. Maron MS, Olivotto I, Zenovich AG, et al., Hypertrophic cardiomyopathy is predominantly a disease of left ventricular outflow tract obstruction, Circulation, 2006;114:2232-9.

56. Olivotto I, Maron MS, Autore C, et al., Assessment and significance of left ventricular mass by cardiovascular magnetic resonance in hypertrophic cardiomyopathy, J Am Coll Cardiol, 2008;52:559-66.

57. Moon JC, Reed E, Sheppard MN, et al., The histologic basis of late gadolinium enhancement cardiovascular magnetic resonance in hypertrophic cardiomyopathy, I Am Coll Cardiol, 2004;43:2260-4.

58. Moon JC, McKenna WJ, McCrohon JA, et al., Toward clinica risk assessment in hypertrophic cardiomyopathy with gadolinium cardiovascular magnetic resonance, J Am Coll Cardiol, 2003;41:1561-7.

59. O'Hanlon $\mathrm{R}$, Grasso A, Roughton $\mathrm{M}$, et al., Prognostic significance of myocardial fibrosis in hypertrophic cardiomyopathy, J Am Coll Cardiol, 2010:56:867-74.

60. Green JJ, Berger JS, Kramer CM, et al., Prognostic value of late gadolinium enhancement in clinical outcomes for hypertrophic cardiomyopathy, JACC Cardiovasc Imaging, 2012:5:370-7.

61. Lyons KS, Dixon LJ, Johnston N, et al., Late gadolinium 
enhancement is common in patients with hypertrophic cardiomyopathy and no clinical risk factors for sudden cardiac death: A single center experience, Cardiol J. 2014:21:29-32.

62. Bruder O, Wagner A, Jensen CJ, et al., Myocardial scar visualized by cardiovascular magnetic resonance imaging predicts major adverse events in patients with hypertrophic cardiomyopathy, J Am Coll Cardiol, 2010;56:875-87.

63. Ismail TF, Jabbour A, Gulati A, et al., Role of late gadolinium enhancement cardiovascular magnetic resonance in the risk stratification of hypertrophic cardiomyopathy. Heart 2014;100:1851-8.

64. Ho CY, Abbasi SA, Neilan TG, et al., T1 measurements identify extracellular volume expansion in hypertrophic cardiomyopathy sarcomere mutation carriers with and without left ventricular hypertrophy, Circ Cardiovasc Imaging, 2013;6:415-22.

65. Ho CY, López B, Coelho-Filho OR, et al., Myocardial fibrosis a an early manifestation of hypertrophic cardiomyopathy, N Engl J Med, 2010;363:552-63.

66. Kwon DH, Smedira NG, Rodriguez ER, et al., Cardiac magnetic resonance detection of myocardial scarring in hypertrophic cardiomyopathy: correlation with histopathology and
prevalence of ventricular tachycardia, I Am Coll Cardiol, 2009:54:242-9.

67. Kwon DH, Setser RM, Popović ZB, et al., Association of myocardial fibrosis, electrocardiography and ventricula tachyarrhythmia in hypertrophic cardiomyopathy: a delayed contrast enhanced MRI study, Int I Cardiovasc Imaging, 2008;24:617-25

68. Adabag AS, Maron BJ, Appelbaum E, et al., Occurrence and frequency of arrhythmias in hypertrophic cardiomyopathy in relation to delayed enhancement on cardiovascular magnetic resonance, J Am Coll Cardiol, 2008:51:1369-74.

69. Olivotto I, Maron BJ, Appelbaum E, et al., Spectrum and clinical significance of systolic function and myocardial fibrosis assessed by cardiovascular magnetic resonance in hypertrophic cardiomyopathy, Am I Cardiol, 2010;106:261-7.

70. Chan RH, Maron BJ, Olivotto I, et al., Prognostic value of quantitative contrast-enhanced cardiovascular magnetic resonance for the evaluation of sudden death risk in patients with hypertrophic cardiomyopathy, Circulation, 2014;130:484-95.

71. McKenna WJ, Nagueh SF, Cardiac magnetic resonance imaging and sudden death risk in patients with hypertrophic cardiomyopathy, Circulation, 2014:130:455-7.

72. Dass S, Suttie JJ, Piechnik SK, et al., Myocardial tissue characterization using magnetic resonance noncontrast t mapping in hypertrophic and dilated cardiomyopathy, Circ Cardiovasc Imaging, 2012;5:726-33.

73. MCGill LA, ISmail TF, Nielles-Vallespin S, et al., Reproducibility of in-vivo diffusion tensor cardiovascular magnetic resonance in hypertrophic cardiomyopathy, I Cardiovasc Magn Reson, 2012:14:86.

74. Wong TC, Cardiovascular Magnetic Resonance Imaging of Myocardial Interstitial Expansion in Hypertrophic Cardiomyopathy, Curr Cardiovasc Imaging Rep, 2014;7:9267.

75. Ellims AH, lles $L M$, Ling LH, et al., A comprehensive evaluation of myocardial fibrosis in hypertrophic cardiomyopathy with cardiac magnetic resonance imaging: linking genotype with fibrotic phenotype, Eur Heart I Cardiovasc Imaging, 2014; 15:1108-16

76. Arad M, Monserrat L, Haron-Khun S, et al., Merits and pitfalls of genetic testing in a hypertrophic cardiomyopathy clinic ISr Med Assoc J, 2014:16:707-13.

77. Calore C, De Bortoli M, Romualdi C, et al., A founder MYBPC3 mutation results in HCM with a high risk of sudden death after the fourth decade of life, J Med Genet, 2015;52:338-47.

78. Gimeno JR, Monserrat L, Pérez-Sánchez I, et al., Hypertrophic cardiomyopathy. A study of the troponin-T gene in 127 Spanish families, Rev Esp Cardiol, 2009;62:1473-7.

79. Revera M, Van der Merwe L, Heradien M, et al., Long-term follow-up of R403WMYH7 and R92WTNNT2 HCM families: mutations determine left ventricular dimensions but not wall thickness during disease progression, CardiovasC $J$ Afr, 2007;18:146-53.

80. Maron BJ, Roberts WC, Arad M, et al., Clinical outcome and phenotypic expression in LAMP2 cardiomyopathy, JAMA, 2009;301:1253-9.

81. Maron BJ, Maron MS, Semsarian C, Double or compound sarcomere mutations in hypertrophic cardiomyopathy: a potential link to sudden death in the absence of convention risk factors, Heart Rhythm, 2012;9:57-63.

82. Ingles J, Doolan A, Chiu C, et al., Compound and double mutations in patients with hypertrophic cardiomyopathy: implications for genetic testing and counselling, I Med Genet, 2005; $42:$ :e59.

83. Wang J, Wang Y, Zou Y, et al., Malignant effects of multiple rare variants in sarcomere genes on the prognosis of patients with hypertrophic cardiomyopathy, Eur J Heart Fail, 2014;16:950-7.

84. Maron MS, Finley JJ, Bos JM, et al., Prevalence, clinical significance, and natural history of left ventricular apical aneurysms in hypertrophic cardiomyopathy, circulation, 2008:118:1541-9.

85. Harris KM, Spirito P, Maron MS, et al., Prevalence, clinical profile, and significance of left ventricular remodeling in the end-stage phase of hypertrophic cardiomyopathy, circulation 2006;114:216-25

86. Kawarai H, Kajimoto K, Minami Y, et al., Risk of sudden death in end-stage hypertrophic cardiomyopathy, I Card Fail, 2011;17:459-64.
87. Femenía F, Arce M, Van Grieken J, et al., Fragmented QRS in Hypertrophic Obstructive Cardiomyopathy (FHOCM) Study Investigators. Fragmented QRS as a predictor of arrhythmic events in patients with hypertrophic obstructive cardiomyopathy, I Interv Card Electrophysiol, 2013;38:159-65

88. Kang $\mathrm{KW}$, Janardhan $\mathrm{AH}$, Jung $\mathrm{KT}$, et al., Fragmented QRS as a candidate marker for high-risk assessment in hypertrophic cardiomyopathy, Heart Rhythm, 2014;11:1433-40.

89. Minami $Y$, Kajimoto $K$, Terajima $Y$, et al., Clinical implications of midventricular obstruction in patients with hypertrophic cardiomyopathy, J Am Coll Cardiol, 2011;57:2346-55.

90. Efthimiadis GK, Pagourelias ED, Parcharidou D, et al., Clinical characteristics and natural history of hypertrophic cardiomyopathy with midventricular obstruction, Circ $\lrcorner$ 2013;77:2366-74

91. Eriksson MJ, Sonnenberg B, Woo A, et al., Long-term outcome in patients with apical hypertrophic cardiomyopathy, I Am Coll Cardiol, 2002;39:638-45.

92. Sorajja P, Ommen SR, Nishimura RA, et al., Adverse prognosis of patients with hypertrophic cardiomyopathy who have epicardial coronary artery disease, Circulation. 2003;108:2342-8.

93. Maron MS, Olivotto I, Maron BJ, et al., The case for myocardial ischemia in hypertrophic cardiomyopathy, I Am Coll Cardiol, 2009;54:866-75.

94. Petersen SE, Jerosch-Herold M, Hudsmith LE, et al., Evidence for microvascular dysfunction in hypertrophic cardiomyopathy: new insights from multiparametric magnetic resonance imaging, Circulation, 2007;115:2418-25.

95. Gyllenhammar T, Fernlund E, Jablonowski R, et al., Young patients with hypertrophic cardiomyopathy, but not subjects at risk, show decreased myocardial perfusion reserve quantified with CMR, Eur Heart I Cardiovasc Imaging, 2014;15:1350-7.

96. Huang L, Han R, Ai T, Sun Z, et al., Assessment of coronary microvascular dysfunction in hypertrophic cardiomyopathy: first-pass myocardial perfusion cardiovascular magnetic resonance imaging at 1.5 T, Clin Radiol, 2013;68:676-82

97. Peteiro J, Bouzas-Mosquera A, Fernandez X, et al., Prognostic value of exercise echocardiography in patients with hypertrophic cardiomyopathy, I Am SOC Echocardiogr, 2012;25:182-9.

98. Desai MY, Bhonsale A, Patel P, et al., Exercise echocardiography in asymptomatic HCM: exercise capacity, and not LV outflow tract gradient predicts long-term outcomes, JACC Cardiovasc Imaging, 2014;7:26-36.

99. Finocchiaro G, Haddad F, Knowles JW, et al., Cardiopulmonary responses and prognosis in hypertrophic cardiomyopathy: a potential role for comprehensive noninvasive hemodynamic assessment, JACC Heart Fail, 2015;3:408-18. 